\title{
Doing an Excellent Review of a Sex Roles Paper
}

\author{
Irene Hanson Frieze
}

Published online: 13 March 2010

(C) Springer Science+Business Media, LLC 2010

Reviewers do an enormous service for Sex Roles. An excellent review helps the author as well as the Editor, and helps to strengthen the journal. This editorial discusses some issues to consider when doing a review for this journal. It is not essential that a reviewer consider each of these points, but it should be clear what aspects of the paper are being considered in the review and which are not.

First, in writing your review, remember that your review is going to a real person. Be diplomatic! It is very helpful to begin a review by noting the strengths of the manuscript before moving on to the criticisms. Reviewers have read an article looking for possible problems, so it is helpful to step back before writing the review and think about how this feedback is best presented.

Second, note whether the paper is an initial submission or a revision. Issues addressed in the reviewer comments depend on the stage of revision. For an initial review of a paper, focus on the overall theory, measures and analyses. Save specific suggestions about how sentences might be more clearly worded for later reviews. When looking at a revision, be sure to comment on whether or not the author responded to earlier feedback on the paper.

Third, it is not necessary to provide line by line spelling or formatting corrections. A general sentence explaining that the paper does not follow APA style guidelines or that the paper contains many spelling errors is sufficient.

Here are points that might be considered in your review:

1. Topic. It is helpful to briefly review the basic topic and research questions at the beginning of the review. Consider issues such as: Is the topic appropriate for Sex

I. H. Frieze $(\bowtie)$

University of Pittsburgh,

Pittsburgh, PA, USA

e-mail: frieze@pitt.edu
Roles, and would the research questions be of interest to the readers of the journal? Does the topic add to existing research or does it merely replicate work already done? Sex Roles is a journal that has consideration of gender roles and gender differences as a central focus. Any article to be published in this journal needs to consider gender as the major topic to be examined.

2. Hypotheses or Research Questions. Does the Introduction include a set of clearly articulated research questions or hypotheses? Are hypotheses testable? [Any null hypotheses should be noted and should be omitted from the paper].

3. Review of related published research. Is current and relevant work reviewed? Are any key studies omitted? What does this paper potentially add to this existing work? Does the review make it clear to the reader why the predictions being tested in the paper make sense and provide a rationale for the predictions?

4. Methods used. Is the sample clearly described and appropriate for the research questions being asked? Is the sample large enough to provide good statistical power for the proposed analyses? Are the procedures used for data collection clearly described? Are there any obvious biases created by the procedures used? Is the measurement of all variables later included in the Results clearly described? Are appropriate psychometric properties of scales described? This should include the origin of the items, score creation, and Cronbach alphas. Are coding procedures for qualitative data clearly described?

5. Results. Are the analyses what was expected given the hypotheses?

6. Discussion. Does this section link back to the basic theory from the Introduction? Is the focus on the obtained data in the study?

7. Other. Are tables and figures clear? 